\section{(6) OPEN ACCESS}

\title{
MicroRNA-21 is a potential link between non-alcoholic fatty liver disease and hepatocellular carcinoma via modulation of the HBP1-p53-Srebp1c pathway
}

\author{
Heng Wu, Raymond $\mathrm{Ng}_{1}{ }^{2}$ Xin Chen, ${ }^{3}$ Clifford J Steer, ${ }^{1,4}$ Guisheng Song ${ }^{1}$
}

- Additional material is published online only. To view please visit the journal online (http://dx.doi.org/10.1136/ gutjnl-2014-308430).

${ }^{1}$ Department of Medicine, University of Minnesota Medical School, Minneapolis, Minnesota, USA

${ }^{2}$ Agency for Science Technology and Research, Singapore, Singapore ${ }^{3}$ Department of Bioengineering and Therapeutic Sciences, University of California San Francisco, San Francisco, California, USA

${ }^{4}$ Department of Genetics, Cell Biology and Development, University of Minnesota, Minneapolis, Minnesota, USA

\section{Correspondence to} Dr Guisheng Song, Division of Gastroenterology, Hepatology and Nutrition, Department of Medicine, University of Minnesota Medical School, MMC 36, VFW Cancer Research Center, V354, 406 Harvard Street SE,

Minneapolis, MN 55455, USA; gsong@umn.edu

Received 19 September 2014 Revised 12 July 2015 Accepted 14 July 2015 Published Online First 17 August 2015

\section{SLinked}

- http://dx.doi.org/10.1136/ gutjnl-2015-310044

\begin{tabular}{l} 
To cite: Wu H, Ng R, \\
Chen $X_{\text {, et al. Gut }}$ \\
2016;65:1850-1860. \\
\hline
\end{tabular}

\section{ABSTRACT}

Background Non-alcoholic fatty liver disease (NAFLD) is a major risk factor for hepatocellular carcinoma (HCC). However, the mechanistic pathways that link both disorders are essentially unknown.

Objective Our study was designed to investigate the role of microRNA-21 in the pathogenesis of NAFLD and its potential involvement in HCC.

Methods Wildype mice maintained on a high fat diet (HFD) received tail vein injections of microRNA-21-antisense oligonucleotide (ASO) or miR-21 mismatched ASO for 4 or 8 weeks. Livers were collected after that time period for lipid content and gene expression analysis. Human hepatoma HepG2 cells incubated with oleate were used to study the role of miR-21 in lipogenesis and analysed with Nile-Red staining. microRNA-21 function in carcinogenesis was determined by soft-agar colony formation, cell cycle analysis and xenograft tumour assay using HepG2 cells.

Results The expression of microRNA-21 was increased in the livers of HFD-treated mice and human HepG2 cells incubated with fatty acid. MicroRNA-21 knockdown in those mice and HepG2 cells impaired lipid accumulation and growth of xenograft tumour. Further studies revealed that $H b p 1$ was a novel target of microRNA-21 and a transcriptional activator of $p 53$. It is well established that p53 is a tumour suppressor and an inhibitor of lipogenesis by inhibiting Srebp1c. As expected, microRNA-21 knockdown led to increased HBP1 and p53 and subsequently reduced lipogenesis and delayed G1/S transition, and the additional treatment of HBP1siRNA antagonised the effect of microRNA-21-ASO, suggesting that $H B P 1$ mediated the inhibitory effects of microRNA-21-ASO on both hepatic lipid accumulation and hepatocarcinogenesis. Mechanistically, microRNA-21 knockdown induced p53 transcription, which subsequently reduced expression of genes controlling lipogenesis and cell cycle transition. In contrast, the opposite result was observed with overexpression of microRNA-21, which prevented p53 transcription.

Conclusions Our findings reveal a novel mechanism by which microRNA-21, in part, promotes hepatic lipid accumulation and cancer progression by interacting with the Hbp 1-p53-Srebp1c pathway and suggest the potential therapeutic value of microRNA-21-ASO for both disorders.

\section{INTRODUCTION}

The incidence of hepatocellular carcinoma (HCC) worldwide nearly matched its mortality,

\section{Significance of this study}

What is already known on this subject?

- miR-21 is upregulated in human hepatocellular carcinoma.

- p53 is a transcriptional repressor of Srebp1c.

What are the new findings?

- miR-21 is highly expressed in hepatocytes, and its expression is significantly increased in livers of dietary obese mice and human HepG2 cells incubated with fatty acid.

- Antagonising miR-21 in liver prevents hepatic lipid accumulation and growth of xenograft tumour.

- miR-21 knockdown prevents G1/S transition and cancer cell proliferation.

- $H B P 1$ is a novel target of miR-21 and a transcriptional activator of $p 53$.

- HBP1 mediates the inhibitory effects of miR-21-anti-sense oligonucleotide on hepatic lipid accumulation and hepatocarcinogenesis.

- miR-21 is a potential association between non-alcoholic fatty liver disease (NAFLD) and hepatocellular carcinoma (HCC) via interacting with the Hbp1-p53-Srebp1c pathway.

How might it impact on clinical practice in the foreseeable future?

- Our data suggest that miR-21 is a potential therapeutic target for both NAFLD and HCC.

demonstrating the aggressive nature of this malignancy and limited therapeutic options. ${ }^{1}$ Although $\mathrm{HBV}$ and HCV are major risk factors of HCC, nonalcoholic fatty liver disease (NAFLD) remains a common underlying pathology to the majority of patients with HCC in the Western world. ${ }^{2}$ The incidence of NAFLD is growing rapidly due to the prevalence of obesity. ${ }^{3}$ It is estimated that $90 \%$ of obese patients have some form of fatty liver, ranging from simple steatosis to more severe forms of non-alcoholic steatohepatitis (NASH) and cirrhosis with its associated high risk of HCC. In addition, given limited effects of chemotherapy and the relative insensitivity of HCC to radiotherapy, complete tumour extirpation represents the only choice for a long-term cure. Unfortunately, the 
majority of patients are not eligible for surgical resection because of tumour extent or underlying liver dysfunctions including NAFLD. As described above, despite the strong association between NAFLD and HCC, the underlying mechanisms are largely unknown due in part to their complex nature of disease.

The discovery of a class of naturally occurring small noncoding RNAs, termed microRNAs (miRNAs), ${ }^{4}$ has stimulated a new field of research on NAFLD and HCC. Alterations in miRNA expression have been reported in human individuals with NAFLD/NASH and HCC. ${ }^{6}{ }^{7}$ Reflective of their key roles in lipid metabolism and carcinogenesis, ${ }^{5} 8$ miRNAs have been suggested as novel therapeutic targets for both metabolic diseases and human cancers. However, the miRNAs associated with both NAFLD and its potential sequel HCC are poorly described. Our interest in miR-21 arose initially from hepatocyte-specific miRNA profiling studies in mouse livers, in which we showed that miR-21 is highly expressed in hepatocytes. Furthermore, we observed that high fat diet (HFD) treatment significantly induced expression of miR-21 in livers of mice. By antagonising miR-21 in liver, we were able to prevent hepatic lipid accumulation in dietary obese mice. Consistent with our findings, miR-21 expression was significantly upregulated in human patients with NASH. ${ }^{6}$ It is also known that miR-21 is a potent promoter of HCC and other human cancers. ${ }^{7} 9$ These data led us to hypothesise that miR-21 plays an important role in the pathogenesis of NAFLD and its potential progression to HCC. In the present study, we have investigated the regulatory role of miR-21 in linking NAFLD and $\mathrm{HCC}$ in both in vivo and in vitro model systems.

\section{MATERIALS AND METHODS Bioinformatic analysis}

Identification of miR-21 target genes was conducted as previously described with minor revision. ${ }^{10}$ In detail, we compiled a list of downregulated genes in livers of patients with NAFLD/ NASH by downloading their microarray data from GEO (http:// www.ncbi.nlm.nih.gov/geo/). ${ }^{11}$ mRNA profiles of six normal liver samples (male) and eight NAFLD/NASH liver samples (male) were compared using GeneSpring (Agilent Technologies Genomics). Differentially expressed genes were defined by a log-scale ratio $\leq 0.3$ between paired samples with a $\mathrm{p}<0.05$. Based on these criteria, we identified 1219 downregulated probes in NAFLD/NASH samples (see online supplementary table S1). To identify genes with binding motifs for miR-21, we downloaded the target gene databases of miR-21 based on TargetScan, ${ }^{12}$ Pictar $^{13}$ and Starbase. ${ }^{14}$ Only hits from Target or PicTar algorithm that were confirmed by Ago HITS-CLIP (highthroughput sequencing of RNAs isolated by cross-linking immunoprecipitation (HITS-CLIP) from Argonaute protein complex) were selected. These three databases were compared using Microsoft Access 2000, yielding 219 potential targets that have miR-21 binding motif (see online supplementary table S2). We then compared 1219 downregulated probes in livers of patients with NAFLD/NASH with 219 genes that have at least one binding motif for miR-21 using Microsoft Access 2010, which resulted in an overlap of 13 genes between two databases that were considered as potential targets of miR-21 (see online supplementary table S3). Gene ontology (GO) analysis was done using PathwayStudio software (Elsevier).

Animal, diet treatment and sample collection Male Dicer $1^{\text {fl/fl }}$ mice on a mixed 129S4, C57Bl/6 strain background ${ }^{15}$ were crossed with $\mathrm{C} 57 \mathrm{Bl} / 6 \mathrm{Alb}-\mathrm{Cre}^{+/-}$mice $^{16}$ to generate Dicer1 $1^{f l f l}$, Alb-Cre $e^{+/-}$mice (mice are from Dr Holger
Willenbring's lab at the University of California, San Francisco). To specifically investigate the impact of miRNAs on mature liver function, we initiated Cre recombinase expression in 8-week-old to 10 -week-old mice. ${ }^{17}$ To restrict Cre expression to hepatocytes, we used a hepatocyte-specific Transthyretin (Ttr) promoter and pseudotyped the vector genome with capsids from AAV8, a serotype that can transduce virtually all hepatocytes in vivo without causing toxicity. ${ }^{10} 1718$

To determine the effect of hepatic lipid accumulation on miRNA expression, 8-week-old wildtype male C57Bl/6 mice (Jackson Laboratory, $\mathrm{n}=6$ ) were maintained on either a normal chow diet (Open Source D12450B: 10\% Kcal fat) or an HFD (Open Source D12492: 60\% Kcal fat) for 4 weeks as described by Vickers et al. ${ }^{19}$ After 4 weeks of HFD administration, livers were collected for miRNA and gene expression analysis.

To determine the role of miR-21 in NAFLD, we synthesised locked nucleic acid anti-miR-21 anti-sense oligonucleotide (ASO) (Exiqon) specifically targeting miR-21 and also generated miR-21-mismatched-ASO (miR-21-MM-ASO), a control ASO that differs from miRNAs in four mismatched base pairs. The male C57Bl/6 mice kept on HFD for 4 weeks were divided into two groups; one group $(n=8)$ was treated with miR-21-ASO and the other with miR-21-MM-ASO (control, $n=8$ ). Mice received a dose of $25 \mathrm{mg} / \mathrm{kg}$ miR-21-ASO or miR-21-MM-ASO $(0.9 \% \mathrm{NaCl})$ weekly for 4 or 8 weeks via tail vein injection. At those times, the mice were anaesthetised, and blood was collected by way of cardiac puncture. Subsequently, the livers were harvested and immediately frozen in liquid nitrogen and stored at $-80^{\circ} \mathrm{C}$ for gene expression and histological analysis.

\section{Fatty acid treatment of HepG2 cells}

HepG2 cells were obtained from Dr Xin Chen's laboratory at the University of California, San Francisco. Sodium oleate was obtained from Sigma-Aldrich and was dissolved in Dulbecco's modified Eagle medium (DMEM) with 1\% fatty acid free bovine serum albumin (BSA) (Sigma). Oleate treatment of HepG2 cells was carried out as previously described with minor revision. ${ }^{10}{ }^{20}$ Specifically, HepG2 cells were plated in four-well chamber slides with DMEM medium supplemented with 10\% fetal bovine serum (Invitrogen). After 24 h, HepG2 cells were treated with either control medium (DMEM supplemented with $1 \%$ fatty acid free BSA) or medium containing oleate $(0.5 \mathrm{mM})$. The cells were cultured for another $24 \mathrm{~h}$, then lipid accumulation and miR-21 expression were determined by Nile Red Staining (Sigma-Aldrich) and qRT-PCR, respectively (see online supplementary materials and methods for details).

\section{Cell proliferation analysis}

HepG2 cells were transfected with miR-21-ASO, scrambled control or miR-21-ASO plus HBP1-siRNA using lipofectamine 2000 (Invitrogen). After $48 \mathrm{~h}$, cell proliferation was determined using a MTT (3-(4,5-dimethylthiazol-2-yl)-2, 5 -diphenyltetrazoliumbromide) cell proliferation kit (Cell Biolabs) according to the manufacturer's instruction (see online supplementary material and methods for details).

\section{Focus formation assay and flow cytometry analysis}

HepG2 cells were used to determine the effect of miR-21 on focus formation and cell cycle progression (see online supplementary material and methods for details).

\section{Xenograft tumour assay}

Male BALB/c athymic nude mice (6 weeks old) purchased from Jackson Laboratory were used to study the role of miR-21 in 
promoting growth of xenograft tumour from HepG2 cells (see online supplementary material and methods for details).

\section{Histological analysis}

Frozen sections of liver were stained with Oil Red-O staining. Paraformaldehyde-fixed, paraffin-embedded sections of liver were stained with H\&E (see online supplementary material and methods for details).

\section{Lipid and lipoprotein analysis}

Both plasma and hepatic lipid content were enzymatically measured in liver lysates and plasma via a colorimetric assay using a triglyceride assay kit from Roche Diagnostics, according to the manufacturer's protocols (see online supplementary material and methods for details).

\section{Western blot and Q-RT-PCR}

Western blot and qRT-PCR were used to determine expression levels of genes. Primers used for quantitative RT-PCR are listed in online supplementary table S4 (see online supplementary material and methods for details).

\section{Statistical analysis}

Statistical analysis was performed using GraphPad Prism Software. Data derived from cell-line experiments were presented as mean \pm SD and assessed by a two-tailed Student's $t$ test. Statistical difference for cell cycle progression analysis was evaluated using $\chi^{2}$ test. Mann-Whitney test was used to evaluate the statistical significance for mouse experiments. Each experiment was repeated at least three times; and the error bars represent the SD. $\mathrm{p}<0.05$ was considered to be statistically significant.

\section{RESULTS}

miR-21 is robustly induced in livers of mice on HFD and HepG2 cells exposed to high levels of fatty acid

Hepatocytes are the major cells that control lipid metabolism and the primary site of NAFLD and HCC. To investigate the role of miRNAs in both disorders, we compiled hepatocytespecific miRNA profiles by comparing miRNAs expression of livers of hepatocyte-specific Dicer1 knockout (DKO) and wildtype mice (see online supplementary table S5). We observed that miR-21 was the most significantly downregulated miRNA in livers of DKO mice ( $\geq 39$-fold reduced), indicating that hepatocytes represent a main source of its expression in the liver (figure 1A). To assess the role of miR-21 in NAFLD, we fed wildtype $\mathrm{C} 57 \mathrm{Bl} / 6$ mice an HFD (see online supplementary figure S1A-C) and measured its hepatic expression. The results showed that miR-21 had a twofold upregulation in the livers of HFD-treated mice (figure 1B), suggesting its potential role in NAFLD.

We also determined whether fatty acids can increase the expression of miR-21 in human hepatoma HepG2 cells. Oleic acids are the most abundant unsaturated fatty acids in liver triglycerides in both normal subjects and patients with NAFLD. ${ }^{21}$ In this study, HepG2 cells were used because of their increased sensitivity to fat accumulation. ${ }^{21}$ Nile-Red staining revealed that oleic acid treatment led to an increase in intracellular lipids in HepG2 cells (figure 1C, D), which was also associated with upregulation of miR-21 (figure 1E). Taken together, our in vivo and in vitro studies indicated that both HFD treatment of mice and exposure of HepG2 cells to fatty acid were able to induce expression of miR-21.
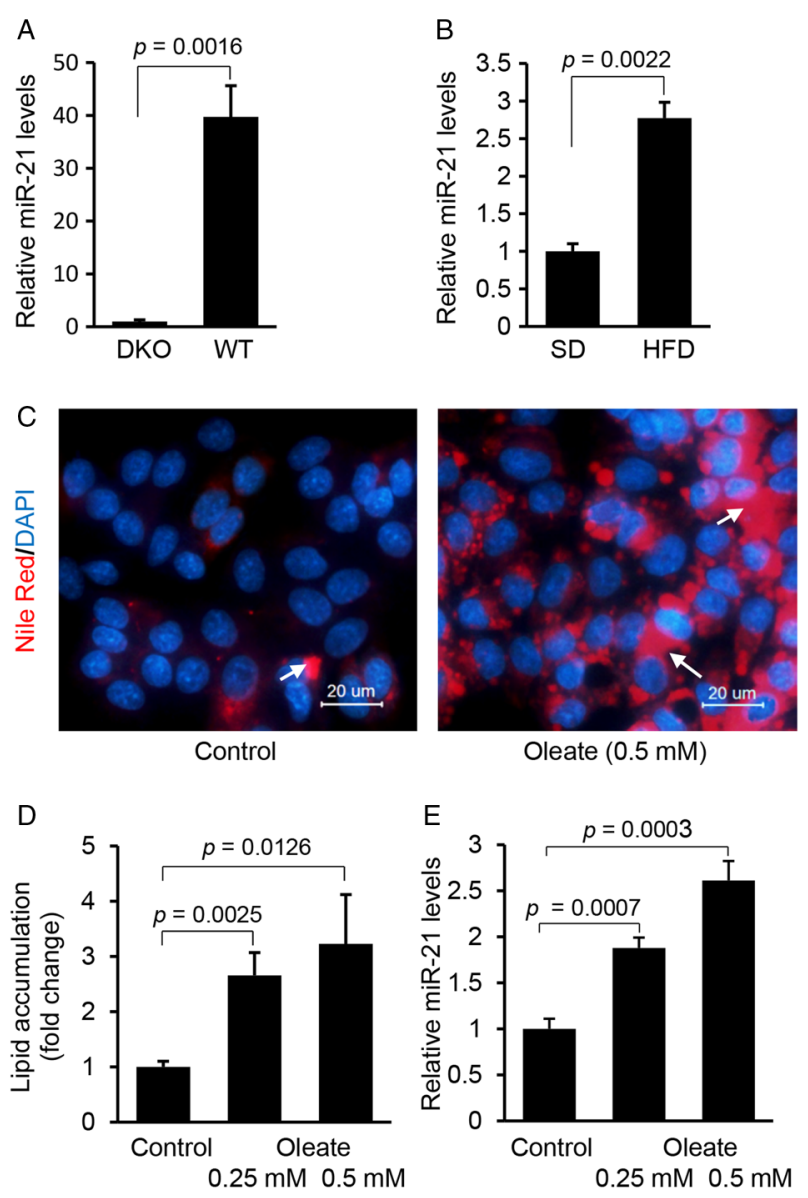

Figure 1 Hepatocyte-specific miR-21 is significantly induced in livers of high fat diet (HFD)-treated mice and HepG2 cells treated with fatty acid. (A) qRT-PCR confirmed that miR-21 was highly expressed in hepatocytes of liver. miR-21 expression in liver was compared between Dicer1 knockout (DKO) $(n=3)$ and wildtype mice $(n=3)$ using qRT-PCR. Data are presented as mean $\pm S D$ ( $p=0.0016$, Student's t test). (B) HFD treatment led to higher levels of miR-21 in livers of mice. Briefly, wildtype mice (C57BI/6) were maintained on HFD for 4 weeks, and then mice were sacrificed and livers collected for miRNA expression analysis. Control mice received standard diet (SD). Data are presented as mean $\pm S D(p=0.0022$, Mann-Whitney test). ( $C$ and D) Oleate treatment increased lipid content and subsequently (E) expression of miR-21 in HepG2 cells. HepG2 cells were maintained in Dulbecco's modified Eagle medium containing $0.5 \mathrm{mM}$ oleate. Data are presented as mean \pm SD. In this multiple-groups experiment, we only performed comparison between two groups and Student's $t$ test was used for statistical analysis. Lipid droplets in human hepatocytes were labelled with arrows.

To further elucidate the role of miR-21 in hepatic lipid accumulation, we began to identify target genes of miR-21 by combining mRNA profiling of livers of NAFLD individuals with the bioinformatic prediction of miR-21 binding motifs within potential target mRNAs. This led us to identify 13 genes including HBP1, SOX7 and RHOB that showed reduced expression in human fatty liver and contained a conserved binding motif for miR-21 (see online supplementary table S3). GO analysis of the above 13 genes revealed that HBP1 was a potent tumour suppressor by preventing G1/S transition of cell cycle. ${ }^{22}{ }^{23}$ In addition, our prediction from in silico algorithms showed that the 3' UTR of HBP1 mRNA was $100 \%$ complementary to the miR-21 $5^{\prime}$ seed region, exhibiting the highest prediction score and binding energy (figure 2A). Therefore, we selected HBP1 as a potential target of miR-21. 
A

Score: 121; Energy: -19.3 kCal/Mol

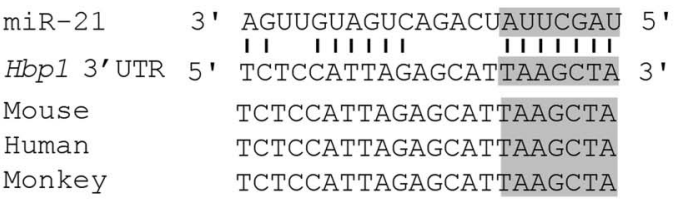

Monkey
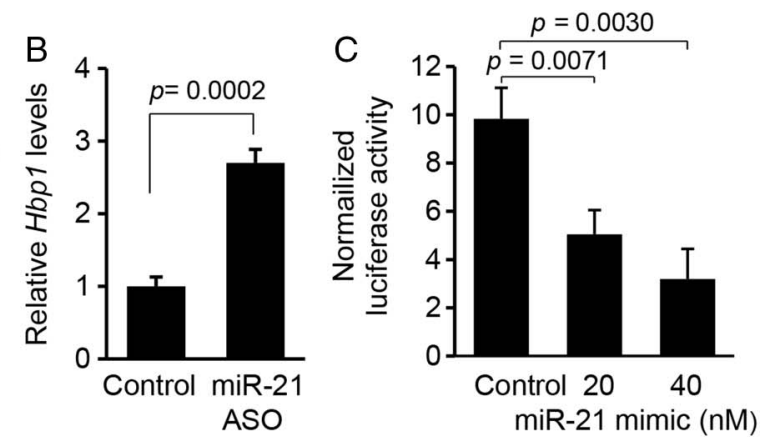

$\mathrm{E}$
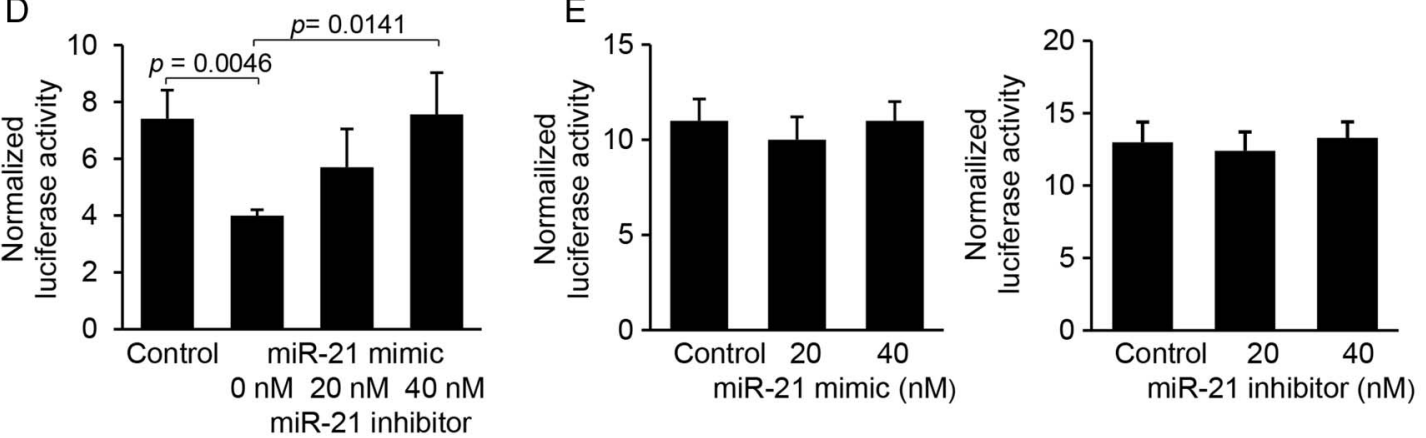

$\mathrm{F}$

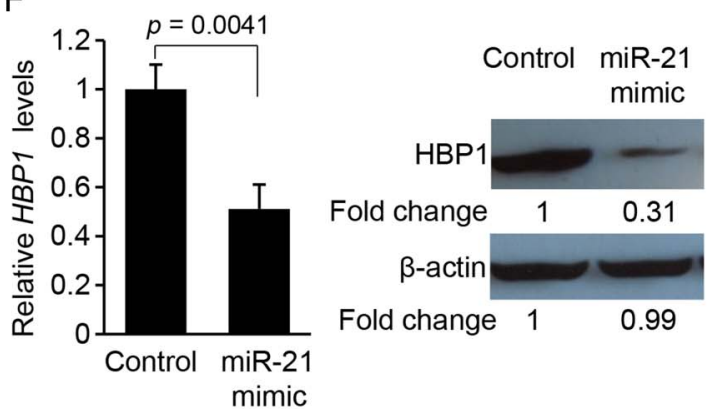

G

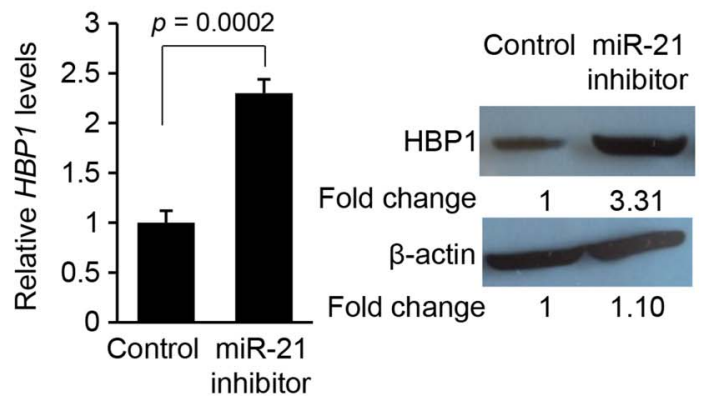

Figure $2 \quad H b p 1$ is a direct target of miR-21. (A) Bioinformatic analysis showing that the seed sequence of miR-21 has a high level of complementarity to Hbp1 $3^{\prime}$ UTR, prediction score and favourable binding energy. Complementary sequences to the seed region of miR-21 within the 3' UTRs of Hbp1 are conserved between human, mouse and monkey (grey highlight). (B) miR-21 knockdown in high fat diet (HFD)-treated mice led to increased $\mathrm{Hbp} 1$ mRNA levels in liver. C57B//6 wildtype mice were kept on normal chow until 8 weeks of age and then maintained on HFD until 16 weeks of age. At 12 weeks of age, the mice were given miR-21-anti-sense oligonucleotide (ASO) $(25 \mathrm{mg} / \mathrm{kg}$, tail vein injection) until 16 weeks of age. C57B//6 mice maintained on HFD and treated with miR-21-MM-ASO served as controls. The expression levels of $H b p 1$ were determined by qRT-PCR. Data are presented as means $\pm S D$ ( $p=0.0002$, Mann-Whitney test). (C) miR-21 mimic transfection into Hepa1, 6 cells caused dose-dependent inhibition of the activity of a luciferase reporter gene linked to the $3^{\prime}$ UTR of mouse Hbp1. Data are presented as mean \pm SD. In this multiple-groups experiment, we only performed comparison between two groups among them and Student's t test was used for statistical analysis. (D) Conversely, transfection with a miR-21 inhibitor antagonised the binding of miR-21 mimics to the 3' UTR of mouse Hbp1, which was reflected by increased luciferase activity. Data are presented as mean \pm SD. Student's $t$ test was used for statistical analysis. (E) Mutated binding motif for miR-21 within Hbp1 3'UTR impaired miR-21 binding, which was reflected by (i) negligible change of luciferase activity after miR-21 mimics treatment; and (ii) miR-21 inhibitor treatment had no effect on luciferase activity. Data are presented as mean $\pm S D$ ( $p \geq 0.1$, Student's $t$ test). (F) Transfection of miR-21 mimics into HepG2 cells inhibited expression levels of endogenous HBP1 as revealed by qRT-PCR and western blot. Data are presented as mean $\pm S D$ ( $p=0.0041$, Student's $t$ test). (G) miR-21 knockdown by transfecting miR-21 inhibitor into HepG2 cells caused an increase in endogenous $H B P 1$ at the protein and mRNA levels. Data are presented as mean $\pm S D(p=0.0002$, Student's $t$ test).

We next determined its expression in the livers of dietary obese mice treated with miR-21-ASO. It was not surprising that Hbp1 expression increased more than twofold in the livers of miR-21-ASO-treated mice compared with those treated with miR-21-MM-ASO (figure 2B). Taken together, hepatic expression of miR-21 was increased in dietary obese mice and livers of human patients with NAFLD/NASH, and Hbp1, as a potential target of miR-21, showed reduced expression in livers of obese mice and human patients with NAFLD (see online supplementary table S3 and figure S1D). Our findings suggested that the crosstalk between miR-21 and Hbp1 might play an important role in hepatic lipid accumulation.

\section{Hbp1 is a direct target of miR-21}

To establish that miR-21 directly recognises the predicted target site within the $3^{\prime}$ UTR of Hbp 1 , the $3^{\prime}$ UTR of mouse Hbp1 mRNA was cloned into a luciferase reporter vector (pMiR-Report) to generate pMiR-Hbp1. Mouse Hepa1,6 cells were transfected with pMiR-Hbp1 and chemically synthesised miR-21 mimic or miR-21 inhibitor. We found that miR-21 mimics significantly downregulated luciferase activity in a dosedependent fashion (figure 2C). Consistently, miR-21 inhibitor antagonised the inhibitory effect of miR-21 mimics on luciferase activity (figure 2D). Furthermore, we mutated the binding motif for miR-21 within the pMiR-Hbp1 $3^{\prime}$ UTR and found that both 
mimics and inhibitors of miR-21 had no effect on luciferase activity (figure $2 \mathrm{E}$ ), indicating a potentially direct interaction between miR-21 and Hbp1 mRNA. To further validate that Hbp1 is a target of miR-21, we increased intracellular levels of miR-21 in HepG2 cells in the absence of fatty acid. qRT-PCR and western blot revealed that miR-21 significantly inhibited expression of HBP1 (figure 2F). In contrast, miR-21 knockdown led to an increase in mRNA and protein levels of HBP1 in HepG2 cells (figure $2 \mathrm{G}$ ). Together, these results confirmed that $H b p 1$ is a direct target of miR-21.

\section{HBP1 inhibits expression of SREBP1C, CCND1 and CCNB1 by activating $p 53$}

HBP1 is a well-described transcriptional repressor that modulates expression of genes involved in cell cycle progression. ${ }^{23}$ Therefore, we overexpressed HBP1 in HepG2 cells and determined the expression levels of genes involved in cell cycle using Human Cell Cycle $\mathrm{RT}^{2}$ Profiler PCR Assay. Interestingly, we observed that $p 53$ was the most upregulated after overexpression of HBP1 (figure 3A). p53 functions as a tumour suppressor and potent inhibitor of lipogenesis by inhibiting transcription of SREBP1C, ${ }^{24-26}$ leading to our hypothesis that miR-21 plays roles in both lipogenesis and carcinogenesis by interacting with the HBP1-p53 pathway.

Overexpression of HBP1 led to increased mRNA levels of p53, implying that HBP1 might activate transcription of $p 53$ by binding to its promoter. Therefore, we cloned the $p 53$ promoter into a luciferase reporter vector (pGL3-Basic) and generated pGL3-p53. Hepa1,6 cells were transfected with pGL3-p53 and HBP1 expression vector. As expected, overexpression of $H B P 1$ induced luciferase activity (figure 3B), and HBP1 knockdown led to decreased luciferase activity (figure $3 \mathrm{C}$ ). Furthermore, HBP1 knockdown impaired expression of endogenous $p 53$ (figure $3 \mathrm{D}$ ), suggesting that $H B P 1$ was able to activate transcription of $p 53$. HBP1 can function as a transcriptional activator by binding to a specific binding motif (GGGATGGG). ${ }^{22}$ However, we did not identify this binding motif within the promoter of $p 53$, signifying that HBP1 might activate transcription of $p 53$ by interacting with other transcription factors that have binding sites within the $p 53$ promoter.

Srebp1c is a transcription factor that activates genes encoding enzymes required for lipid synthesis. ${ }^{27}{ }^{28}$ Considering the role that $p 53$ plays in inhibiting lipogenesis by modulating Srebp $1 c,{ }^{26}$ we cloned the mouse Srepb1c promoter into pGL3-basic vector (pGL3-Srebp1c). As expected, co-transfection of $p 53$ expression vector pGL3-Srebp1c and into Hepa1,6 cells significantly reduced luciferase activity in a dose-dependent fashion (see online supplementary figure S2A), and in contrast, $p 53$ knockdown induced luciferase activity (see online supplementary figure S2B). Furthermore, we observed that overexpression of $p 53$ led to a decrease in endogenous mRNA levels of SREBP1C and its targeted lipogenic genes SCD1 (stearoyl-CoA desaturase-1), GPAT (glycerol 3-phosphate acyltransferase), and FASN (fatty acid synthase), ${ }^{29}$ and genes controlling cell cycle progression including CCNB1 and CCND1 in HepG2 cells (figure 3E). ${ }^{30}$ p53 knockdown led to the opposite effect (figure $3 \mathrm{~F}$ ), underscoring the central role of $p 53$ in modulating the expression of genes involved in lipogenesis and cell cycle progression.

To further determine whether HBP1 prevents transcription of genes associated with lipogenesis and G1/S transition via p53-SREBP1C pathway, we overexpressed HBP1 in HepG2 cells and determined expression levels of $p 53$, SREBP1C, the lipogenic genes and CCNB1 and CCND1. As confirmed by qRT-PCR, HBP1 overexpression led to increased p53, which subsequently prevented expression of SREBP1C and SCD1, FASN and GPAT, as well as CCNB1 and CCND1 (figure 3G). In summary, our findings suggested that HBP1 is able to simultaneously inhibit expression of CCNB1, CCND1 and SREBP1C by modulating $p 53$.

\section{miR-21 prevents expression of $p 53$ but promotes} transcription of SREBP1C by modulating HBP1 expression

We have shown that HBP1 is a target of miR-21, and HBP1 can activate transcription of $p 53$. Meanwhile, p53 is a transcriptional repressor of Srebp1c. ${ }^{25} 31$ Thus, we hypothesised that miR-21 can simultaneously regulate expression of genes involved in lipogenesis and the G1/S transition by modulating the HBP1-p53-SREBP1C pathway. Indeed, overexpression of miR-21 in HepG2 cells inhibited expression of HBP1, which subsequently led to a reduction in $p 53$ and an increase in mRNA levels of CCND1, CCNB1 and SREBP1C, as well as its target genes including SCD1, FASN and GPAT (figure $3 \mathrm{H}$ ), while miR-21 knockdown led to an opposite effect (figure 3I). Our findings indicated that miR-21 is able to modulate expression of genes controlling lipogenesis and cell cycle progression via the p53-SREBP1C pathway.

\section{miR-21 modulates lipid accumulation in HepG2 cells by interacting with the HBP1-p53 pathway}

We then determined whether overexpression of miR-21 can promote lipogenesis. As expected, miR-21 overexpression prevented expression of HBP1 and p53 (figure 4A), which subsequently promoted lipid accumulation in HepG2 cells (figure 4B, C).

To determine loss of function for miR-21 in lipid accumulation, we transfected miR-21 inhibitor into oleate-treated HepG2 cells to knock down upregulated miR-21. Antagonising miR-21 led to a significant increase in HBP1 and $p 53$ (figure 4D), which subsequently prevented lipid accumulation (figure 4E, F). These data demonstrated that miR-21 was sufficient for the downregulation of HBP1 and $p 53$, which subsequently induced lipid accumulation. To further investigate the role of the interaction between miR-21 and HBP1 in hepatic lipid accumulation, we mutated the binding motif for miR-21 within the $3^{\prime}$ UTR of $H b p 1$ in the pMiR-Hbp1 (referred to as pMiR-Hbp1Mu) and introduced the pMiR-Hbp1 or pMiR-Hbp1Mu into oleatetreated HepG2 cells. Since oleate treatment increases miR-21 expression in HepG2 cells, it was expected that it would lead to a decrease of luciferase activity in HepG 2 cells transfected with pMiR-Hbp1 compared with pMiR-Hbp1Mu. In fact, oleate treatment of HepG2 cells transfected with pMiR-Hbp1 resulted in robust repression of luciferase activity compared with pMiR-Hbp1-Mu (figure 4G). Together, our results indicated that HBP1 is a direct target of miR-21 during lipid accumulation in HepG2 cells and the crosstalk of miR-21 with HBP1 and p53 plays an important role in hepatic lipid accumulation.

\section{Inhibitory effect of miR-21-ASO on hepatic lipid accumulation is mediated by $H B P 1$}

To confirm that miR-21 promotes lipogenesis via HBP1, we initially antagonised miR-21 by transfecting miR-21-ASO into HepG2 cells to induce expression of $H B P 1$, and then knocked down the induced HBP1 using HBP1-siRNA. The results showed that miR-21 knockdown increased $p 53$ and HBP1 and reduced lipid content in HepG2 cells, but additional treatment of HBP1-siRNA offset the effect of miR-21-ASO (figure 5A-C), which suggested that $H B P 1$, in part, mediated the inhibitory effect of miR-21 inhibitor on lipid accumulation. To study the 
A

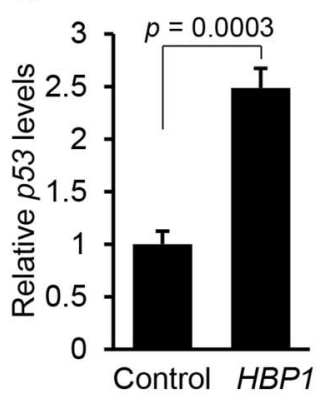

B

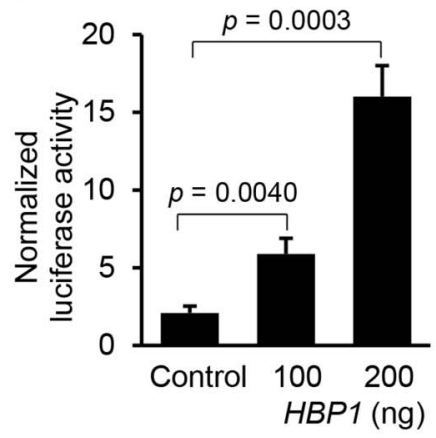

C

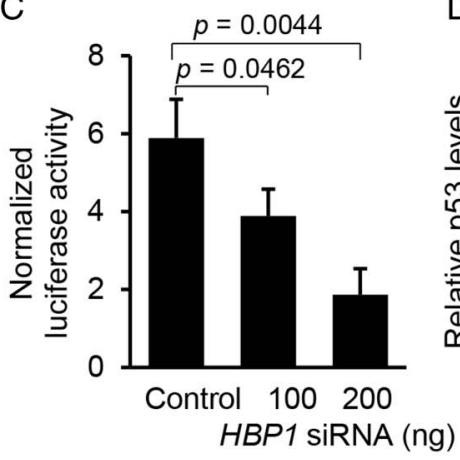

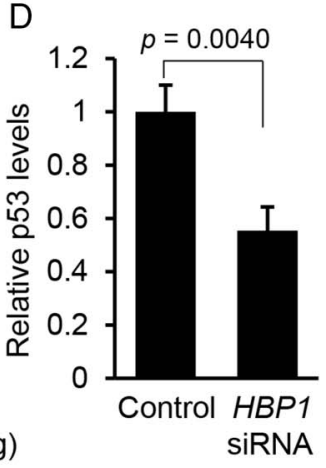

E

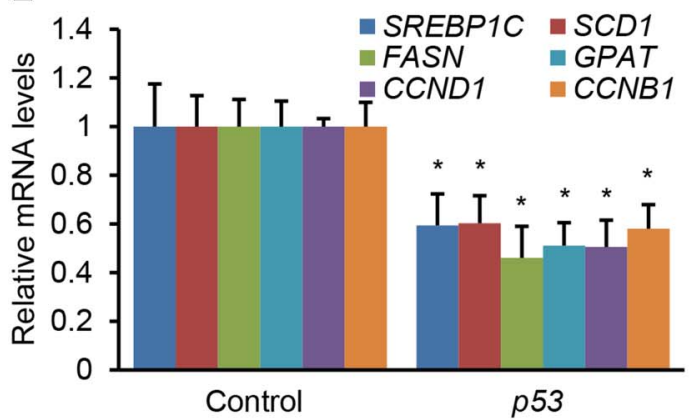

G

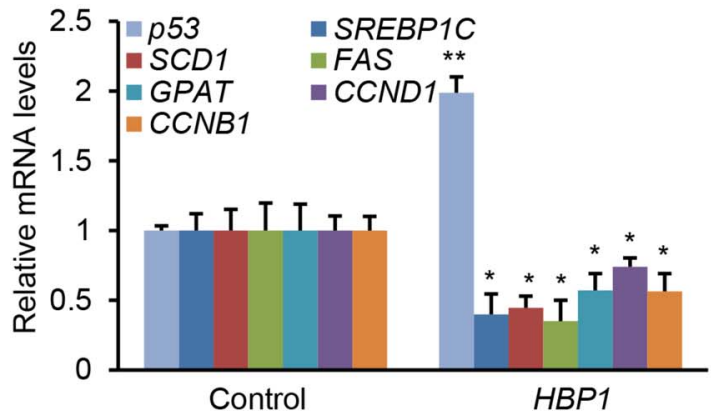

I

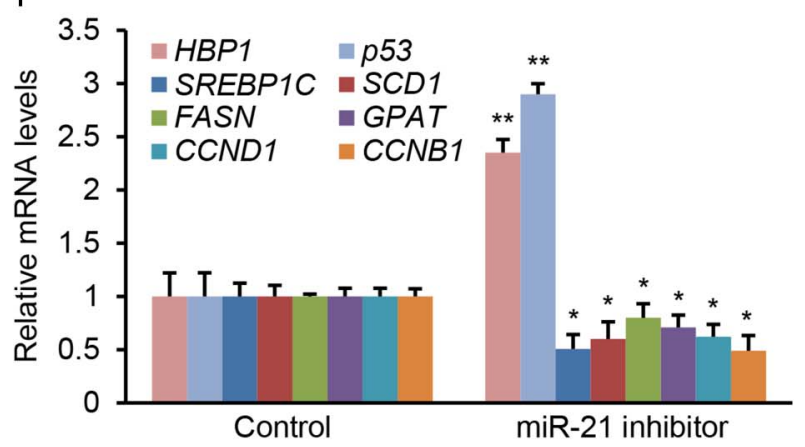

$\mathrm{F}$

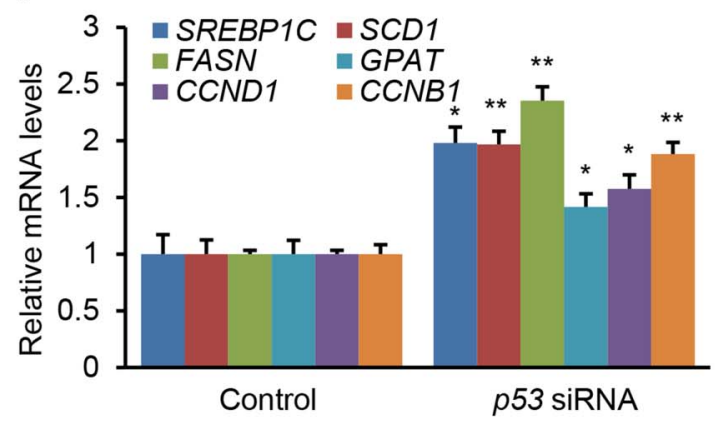

$\mathrm{H}$

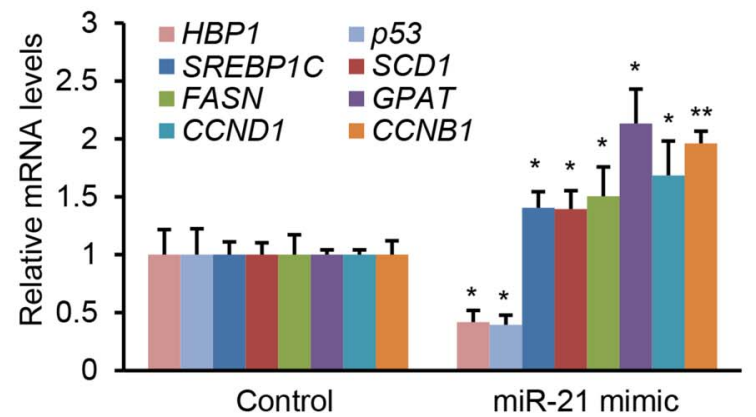

Figure $3 \mathrm{HBP1}$ is a transcriptional activator of $p 53$. (A) Overexpression of HBP1 increased mRNA levels of $p 53$ in human HepG2 cells. Data are presented as means $\pm S D$ ( $p=0.0003$, Student's t test). (B) Overexpression of $H B P 1$ caused dose-dependent increase of the activity of a luciferase reporter gene linked to the $p 53$ promoter. Data are presented as mean \pm SD. Student's t test was used for statistical analysis. (C) HBP1 knockdown via its siRNA decreased luciferase activity driven by $p 53$ promoter. Data are presented as means $\pm S D$. Student's test was used for statistical analysis. (D) HBP1 knockdown via its siRNA resulted in decreased mRNA levels of $p 53$. Data are presented as mean $\pm S D\left({ }^{*} p=0.0040, S t u d e n t ' s\right.$ $t$ test). (E) Overexpression of $p 53$ led to deceased SREBP1C, lipogenic genes SCD1, FASN and GPAT as well as CCND1 and CCNB1 in HepG2 cells. Data are presented as mean $\pm S D\left({ }^{*} p<0.05 ;{ }^{*} p<0.001\right.$; Student's $t$ test). (F) Knockdown of $p 53$ via its siRNA increased mRNA levels of SREBP1C, FASN, SCD1, GPAT, CCND1 and CCNB1 in HepG2 cells. Data are presented as mean $\pm S D\left({ }^{*} p<0.05 ;{ }^{* *} p<0.001\right.$; Student's t test). (G) Overexpression of $H B P 1$ reduced expression of $p 53$, which subsequently led to decreased expression of genes involved in lipogenesis and G1/S transition. Data are presented as mean $\pm S D\left({ }^{*} p<0.05 ;{ }^{* *} p<0.001\right.$; Student's $t$ test). (H) Overexpression of miR-21 inhibited HBP1 and $p 53$, which subsequently promoted expression of the lipogenic genes including SCD1, FASN and GPAT and the genes controlling cell cycle progression including CCNB1 and CCND1. Data are presented as mean $\pm S D\left({ }^{*} \mathrm{p}<0.05 ;{ }^{* *} \mathrm{p}<0.001\right.$; Student's $t$ test). (I) miR-21 knockdown via its inhibitor led to increased $H B P 1$ and $p 53$ and decreased expression of the lipogenic and cell cycle-related genes. Data are presented as mean $\pm S D\left({ }^{*} p<0.05 ;{ }^{* *} p<0.001 ;\right.$ Student's $t$ test). 
Figure 4 MiR-21 modulates lipid accumulation in HepG2 cells by interacting with the $H B P 1-p 53$ pathway. (A) Overexpression of miR-21 inhibited expression of $H B P 1$ and $p 53$, which ( $B$ and $C$ ) subsequently promoted lipid accumulation in HepG2 cells in the presence of $0.25 \mathrm{mM}$ oleate. Data are presented as mean \pm SD. Student's t test was used for statistical analysis. Lipid droplets were labelled with arrows. (D) miR-21 inhibitor transfection into HepG2 cells cultured with the medium containing $0.5 \mathrm{mM}$ oleate led to an increase in $H B P 1$ and $p 53$, which ( $E$ and $F$ ) antagonised the effect of upregulated miR-21 on lipid accumulation. Lipid droplets were labelled with arrows. Data are presented as mean \pm SD. Student's t test was used for statistical analysis. (G) Oleate treatment led to a decrease in luciferase activity of pMiR-Hbp1 as compared with pMiR-Hbp1Mu. Data are presented as mean $\pm S D(p=0.0030$, Student's t test).

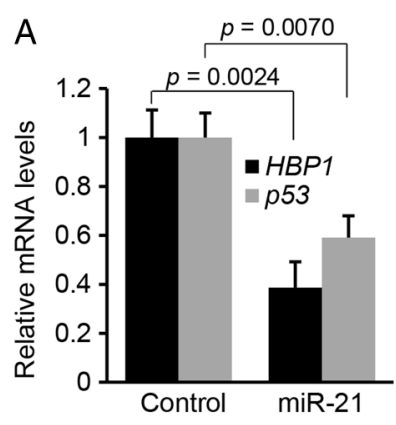

B
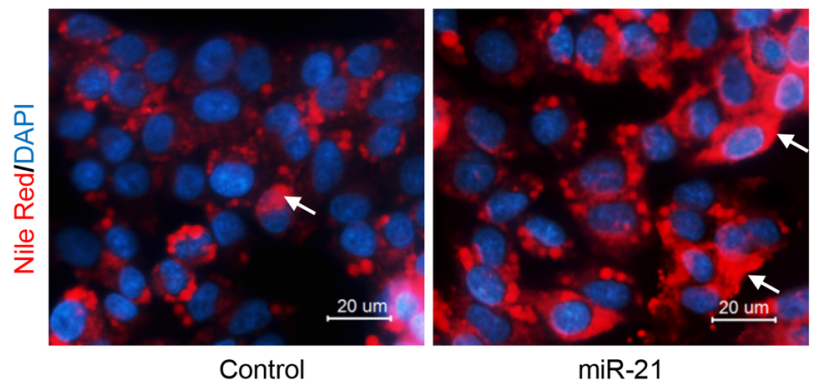

C

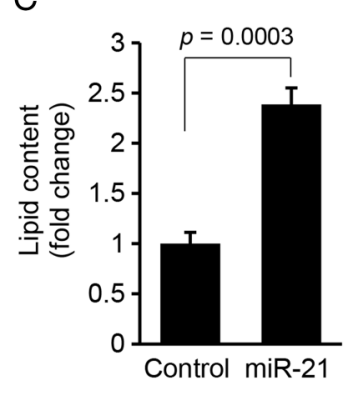

D

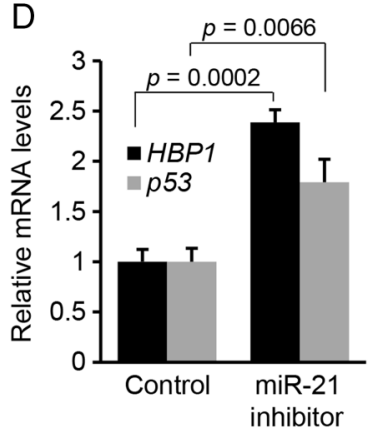

E

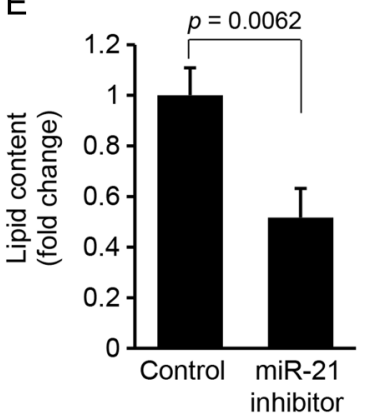

G

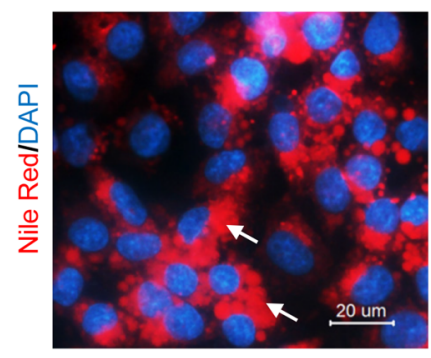

Control

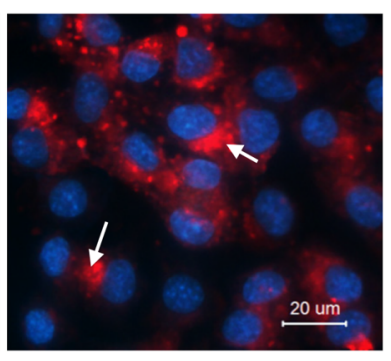

miR-21 inhibitor

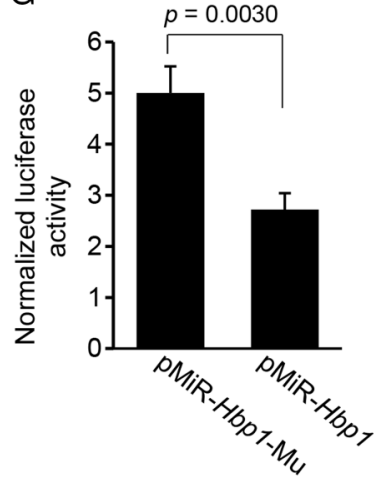

role of the crosstalk between $p 53$ and miR-21 in lipogenesis, we transfected oleated-incubated HepG2 cells with miR-21 mimics or a combination of miR-21 mimics and p53 expression vector. Nile-Red staining and qRT-PCR revealed that miR-21 mimics promoted lipid accumulation in HepG2 cells and the additional p53 overexpression rescued the effect of miR-21 (see online supplementary figure S3A, B). We further determined whether p53 deletion could offset the inhibitory effect(s) of miR-21-ASO on lipogenesis. The results showed that miR-21 knockdown led to decreased lipid content and increased $p 53$, and additional treatment with $p 53$ siRNA offset the effects of miR-21-ASO (see online supplementary figure S3C-E). Taken together, our results indicated that miR-21-induced lipid accumulation is, in part, mediated by HBP1 and $p 53$.

\section{HBP1 mediates the inhibitory effect of miR-21 inhibitor on proliferation, G1/S transition and xenograft tumour from HepG2 cells}

To determine whether $H B P 1$, at least in part, mediates the effects of miR-21 on proliferation and G1/S transition, we adopted a similar strategy as described above. MTT and soft agar colony assays revealed that miR-21-ASO administration caused suppression of cellular proliferation in HepG2 cells, but additional treatment of HBP1-siRNA counteracted the effects of
miR-21-ASO (figure 5D, E). Cell cycle phase distribution of HepG2 cells further showed that miR-21 knockdown led to a significant increase in G1 phase cells and G1/S arrest, but additional treatment of HBP1-siRNA antagonised this effect of miR-21-ASO (figure 5F). Consistent with our in vitro findings, miR-21 knockdown inhibited growth of xenograft tumours in nude mice, and HBP1-siRNA treatment counteracted the effect of miR-21-ASO (figure $5 \mathrm{G}$ ).

As we proposed, $p 53$ is an important mediator of the miR-21-Hbp1-p53 axis. To determine whether $p 53$ mediates the inductive effect of miR-21 on proliferation, we treated HepG2 cells with miR-21 mimic or a combination of miR-21 and $p 53$ expression vector. MTT assay, soft agar colony formation assay and cell cycle analysis revealed that miR-21 promoted proliferation and cell cycle progression, and additional overexpression of $p 53$ offset the inductive effects of miR-21 (see online supplementary figure S4A-E). Furthermore, miR-21-ASO treatment led to reduced proliferation, delayed G1/S transition and repressed growth of xenograft tumour, and the additional treatment of $p 53$ siRNA rescued the inhibitory effects of miR-21-ASO (see online supplementary figure S4F-K). In summary, our findings indicated that HBP1 mediates, at least in part, the inhibitory effects of miR-21-ASO on G1/S transition, proliferation and growth of xenograft tumour. 

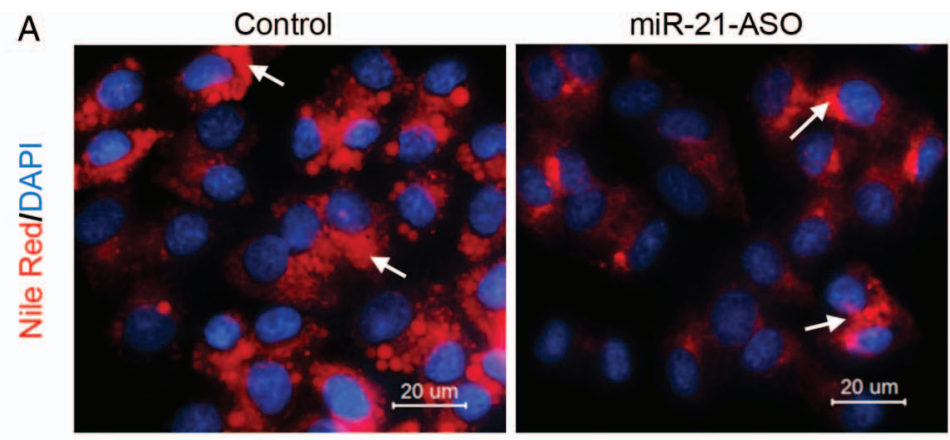

miR-21-ASO + HBP1-siRNA
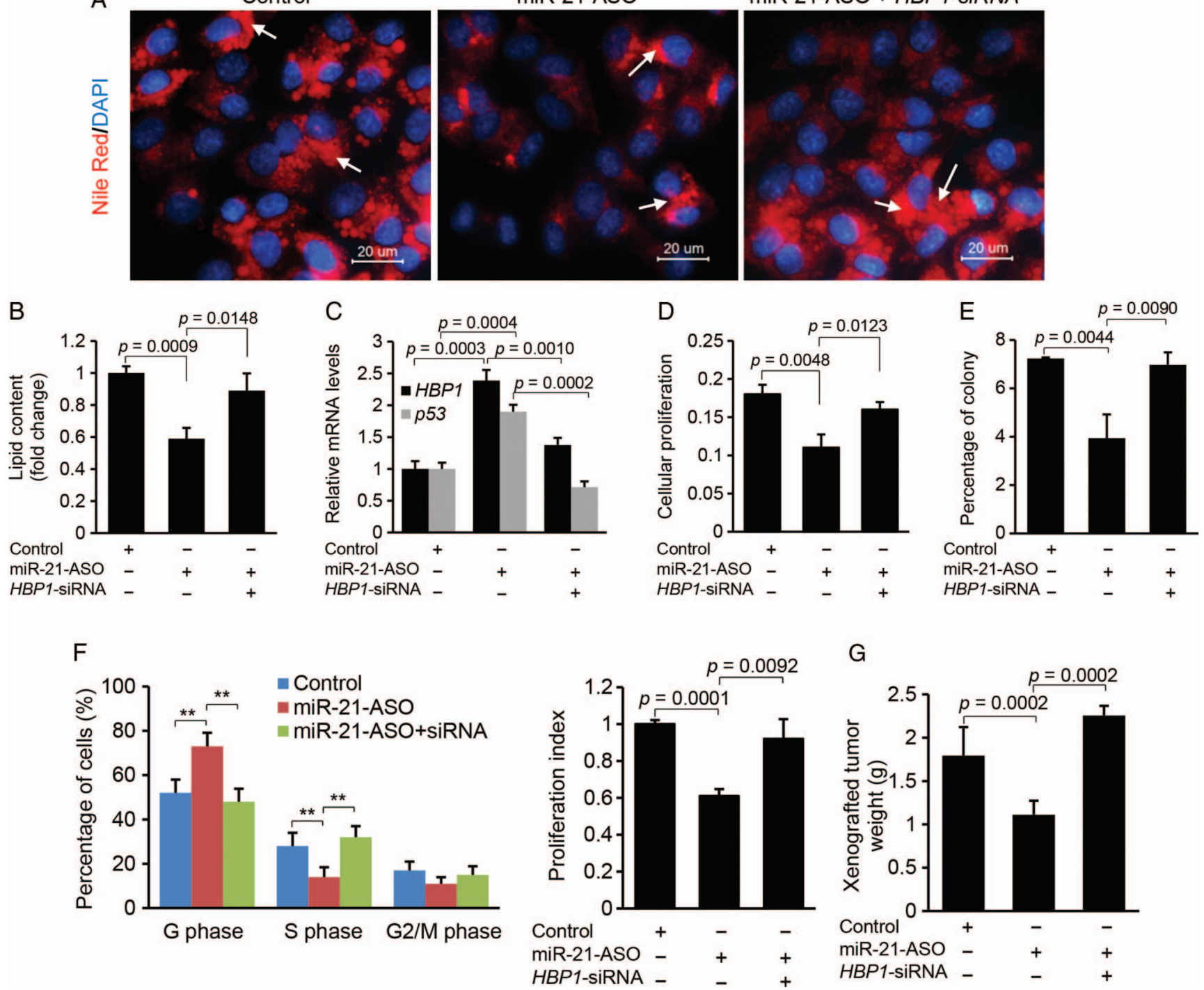

Figure 5 The inhibitory effects of miR-21-anti-sense oligonucleotide (ASO) on lipogenesis, G1/S transition and proliferation are mediated by $H B P 1$. (A and B) miR-21 inhibitor transfection into HepG2 cells reduced lipid content, and additional treatment of HBP1-siRNA restored lipid levels. Lipid droplets were labelled with arrows. Data are presented as mean \pm SD. Student's t test was used for statistical analysis. (C) miR-21 inhibitor treatment induced expression of $H B P 1$ and $p 53$, and additional knockdown of induced $H B P 1$ with its siRNA inhibited expression of $H B P 1$ and $p 53$. Specifically, HepG2 cells were treated with oleate $(0.5 \mathrm{mM})$ to induce miR-21, and then miR-21-ASO was transfected into HepG2 cells to knock down upregulated miR-21. Levels of miR-21, HBP1 and $p 53$ were determined by qRT-PCR. Data are presented as mean \pm SD. Student's $t$ test was used for statistical analysis. (D) MTT assay revealed that antagonising miR-21 via miR-21 inhibitor caused reduced cellular proliferation in HepG2 cells, and additional knockdown of $H B P 1$ by its siRNA rescued the effect of miR-21 inhibitor. Data are presented as mean \pm SD. Student's $t$ test was used for statistical analysis. (E) Soft agar colony formation assay revealed that miR-21 knockdown inhibited the growth of HepG2 cells, and the additional treatment of HBP1-siRNA antagonised the effect of miR-21 inhibitor. Data are presented as mean \pm SD. Student's t test was used for statistical analysis. (F) miR-21-ASO treatment increased the number of cells in the G1 phase but decreased the number of cells in the $S$ phase, and additional knockdown of upregulated HBP1 by its siRNA antagonised the effect of miR-21 inhibitor. Quantification of the cell cycle phase distribution was analysed by flow cytometry. The proliferation index was reduced in the miR-21 inhibitor treated HepG2 cells and the additional treatment of $H B P$-siRNA offset the effect of miR-21 inhibitor. Data are presented as mean $\pm S D\left({ }^{*} p<0.05,{ }^{*}{ }^{*} p<0.001, \chi^{2}\right.$ test). (G) miR-21-ASO inhibited subcutaneous tumours from HepG2 cells in nude mice, and additional treatment of HBP1-siRNA counteracted the effect of miR-21-ASO. HepG2 cells treated with miR-21-MM-ASO, miR-21-ASO or a combination of miR-21-ASO and HBP1-siRNA were injected subcutaneously into different groups of nude mice. Data are presented as mean \pm SD. Student's t test was used for statistical analysis.

Antagonising miR-21 in dietary obese mice prevents hepatic lipid accumulation

Next, we assessed the functional contribution of increased Hbp1 and $p 53$ expression to hepatic lipid accumulation by reducing miR-21 expression in dietary obese mice. C57Bl/6 mice, which had been on an HFD, were injected with either miR-21-ASO or miR-21-MM-ASO for 4 weeks. We observed that antagonising miR-21 significantly reduced levels of triglycerides in livers of
HFD-treated animals (figure 6A, B), in contrast to plasma triglyceride levels (figure 6C). On the other hand, miR-21-ASO treatment had no effect on body and liver weight (see online supplementary table S6). As expected, we also observed a 91\% reduction of hepatic miR-21 expression in mice that received miR-21-ASO compared with miR-21-MM-ASO and a twofold increase of $H b p 1$ and $p 53$ (figure 6D). Four-week treatment of miR-21-ASO showed a strong inhibitory effect on 
Figure 6 Antagonising miR-21 prevented hepatic lipid accumulation in high fat diet (HFD)-treated mice.

( $A$ and $B$ ) miR-21 knockdown inhibited lipid accumulation in livers of HFD-fed mice injected with miR-21-anti-sense oligonucleotide (ASO). Representative images are shown. Lipid droplets in livers are labelled with arrows. Cellular triglyceride content was measured by Oil Red staining and triglyceride content was measured with a triglyceride estimation kit. Data are presented as mean $\pm S D(p<0.0002$, Mann-Whitney test). (C) miR-21 knockdown had no effect on plasma triglyceride in HFD-treated mice. Data are presented as mean $\pm S D(p \geq 0.1$, Mann-Whitney test). (D) miR-21-ASO injection into dietary obese mice resulted in downregulated miR-21 and increased $H b p 1$ and $p 53$ expression. Data are presented as mean \pm SD. Mann-Whitney test was used for statistical analysis. (E) qRT-PCR revealed that HFD-treated mice with decreased levels of miR-21 also retained reduced expression of Scd1, Fasn and Gpat after miR-21-ASO injection. C57BI/6 mice at 8 weeks of age were kept on HFD for an additional 8 weeks. At 12 weeks of age, mice received a dose of $25 \mathrm{mg} / \mathrm{kg}$ miR-21-ASO or miR-21-MM-ASO $(0.9 \%$ $\mathrm{NaCl}$ ) weekly for 4 weeks via tail vein injection. Data are presented as mean ${ }_{ \pm}$SD. Mann-Whitney test was used for statistical analysis.
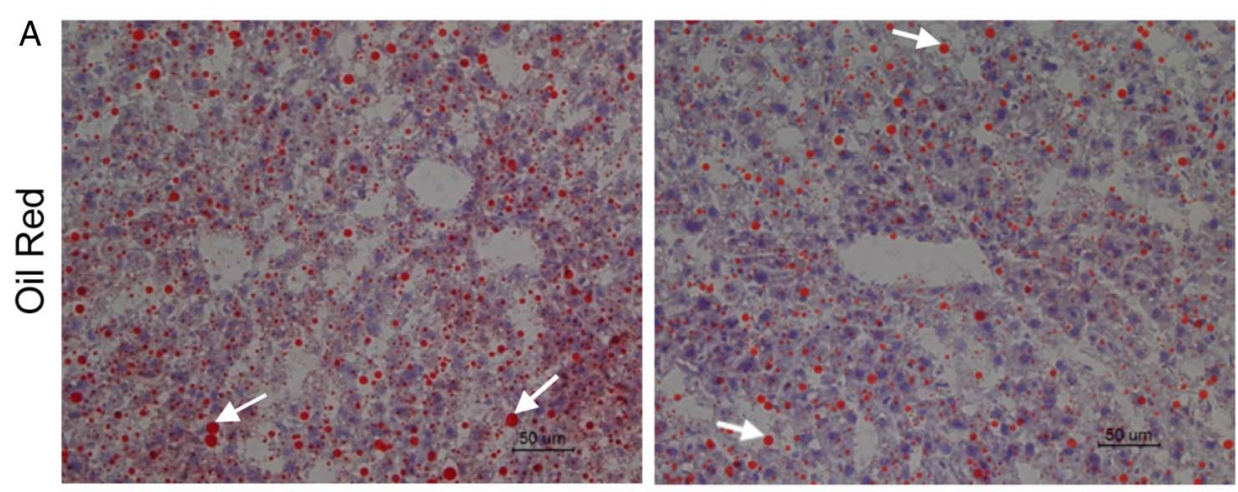

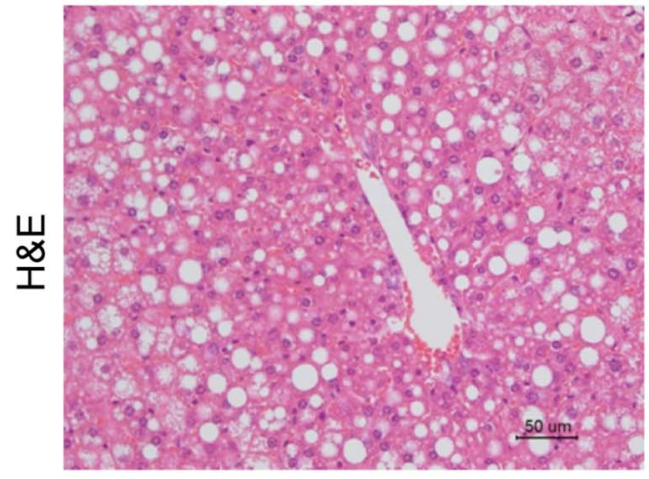

Control
B

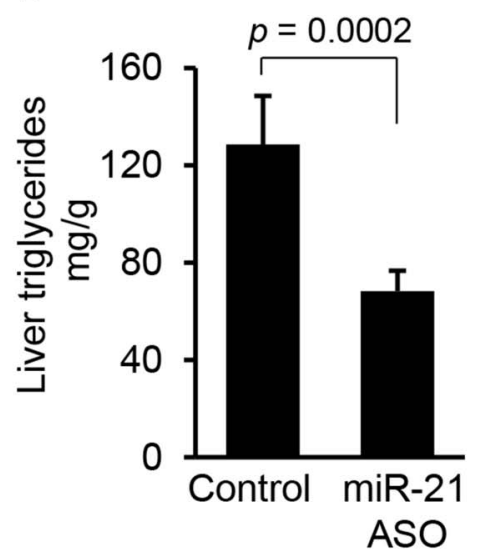

C

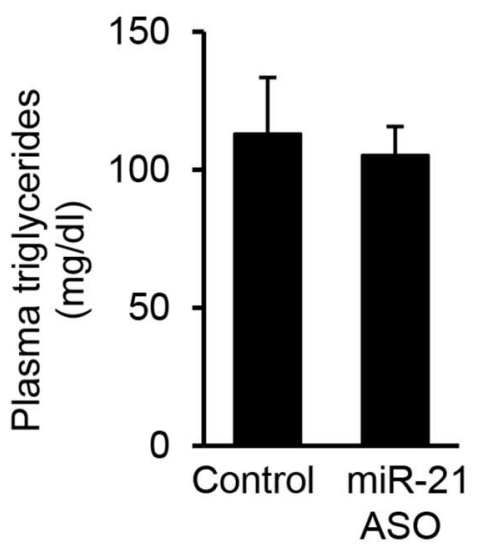

D

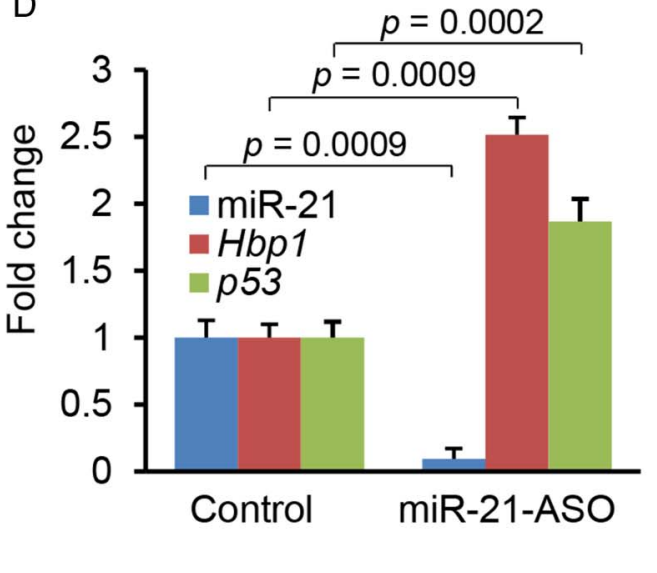

E

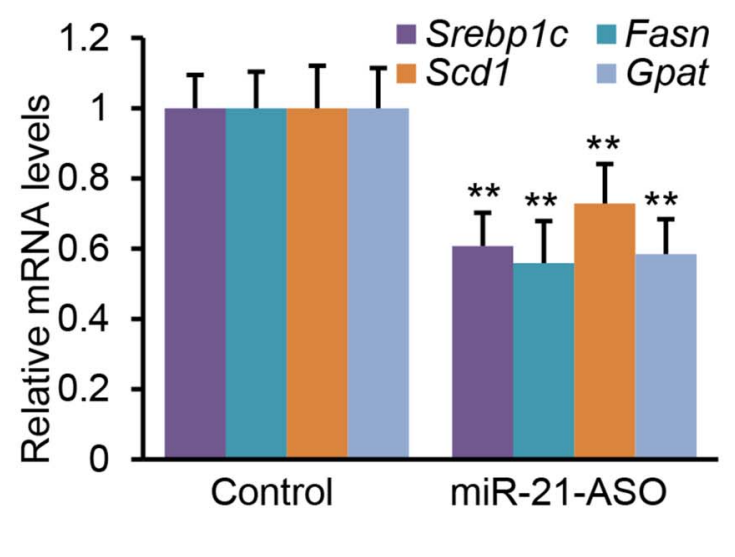


hepatosteatosis, but there were no differences in liver and body weight. Therefore, we increased miR-21-ASO treatment time to 8 weeks, which resulted in decreases in both liver weight (see online supplementary table S7) and hepatic lipid content (see online supplementary figure S5B). However, no difference in body weight (see online supplementary table S7), serum free fatty acid and glycerol still was observed (see online supplementary figure S6A, B). In addition, we also observed that miR-21-ASO treatment had no effect on plasma liver enzymes (see online supplementary table S8). These findings indicated that the crosstalk of miR-21 with $H b p 1$ and $p 53$ plays an important role in hepatosteatosis.

We further compared expression levels of Srebp1c and lipogenic genes in livers of miR-21-ASO and miR-21-MM-ASO treated mice. As expected, miR-21-ASO treatment led to a significant reduction in Srebp1c in the livers of HFD-treated mice (figure 6E). Reduction of Srebp1c due to miR-21 knockdown should impair expression of the lipogenic genes. Indeed, in the miR-21-ASO treated group, the mRNA of three enzymes including $S c d 1$, Fasn and Gpat was downregulated at least 1.5 times those of controls (figure 6E). ${ }^{29}$ Thus, the reduction of Srebp1c was associated with a dramatic reduction in the expression of the target enzymes responsible for lipogenesis, which prevented hepatic lipid accumulation. qRT-PCR also revealed that miR-21 knockdown had no effect on

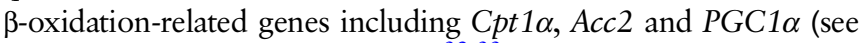
online supplementary figure $\mathrm{S} 6 \mathrm{C}$ ). ${ }^{32} 33$

In summary, our data have shown that HFD treatment led to increased miR-21, decreased $H b p 1$ and $p 53$, which subsequently promoted hepatic lipid accumulation and the potential for HCC, whereas antagonising miR-21 led to the opposite and more therapeutic effect. Hbp1 is inhibited with increased miR-21 levels and its knockdown impaired transcription of $p 53$ by $H b p 1$, which led to reduced $p 53$. The loss of $p 53$ then resulted in increased transcription of Srebp1c, CCNB1 and CCND1, which promoted both lipogenesis and cell replication (figure 7). Our findings indicate the miR-21 plays an important role in linking NAFLD to HCC by interacting with the Hbp1-p53-Srebp1c pathway.

\section{DISCUSSION}

Our study addresses a potentially important role for miR-21 in the development of NAFLD and HCC and defines a novel mechanism by which miR-21 contributes to lipogenesis and

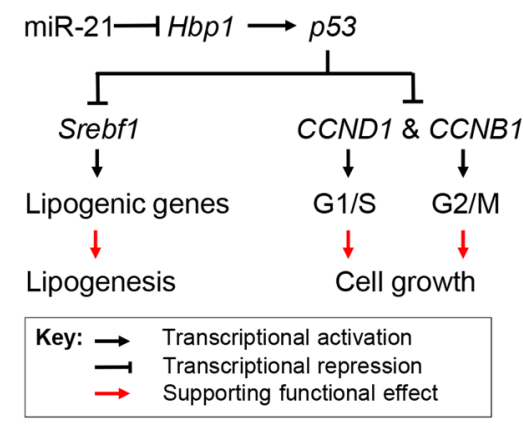

Figure 7 Proposed mechanism by which miR-21 links hepatic lipid accumulation and the development of cancer. Hbp1 is a transcriptional activator of $p 53$, a suppressor of cell cycle progression and inhibitor of lipogenesis by inhibiting transcription of Srebp1c. By directly inhibiting Hbp1 expression, miR-21 prevents expression of $p 53$, which facilitates transcription of genes that are required for lipogenesis and the G1/S transition of cell cycle. As a result, increased miR-21 promotes both hepatic lipid accumulation and potentially carcinogenesis. carcinogenesis via the Hbp1-p53-Srebp1c pathway. The observation that antagonising miR-21 in dietary obese mice potentially improves these metabolic parameters clearly indicates a functional role for increased miR-21 expression in the development of NAFLD. In addition, we also observed that miR-21 knockdown prevented an in situ model of tumorigenesis by targeting HBP1. Increased miR-21 expression is not restricted to murine obesity models of NAFLD and HCC, ${ }^{19}{ }^{34}$ but is also detected in human patients with NASH and HCC. ${ }^{6} 3435$ Thus, miR-21-ASO may act as a unique potential therapeutic approach for the treatment of both disorders.

Despite its putative role in carcinogenesis, ${ }^{36}$ the mechanism by which miR-21 regulates NAFLD is unknown. In this study, we observed that HFD administration resulted in increased miR-21 and its knockdown prevented hepatic lipid accumulation. In addition, we have functionally validated $H b p 1$ as a bona fide target of miR-21, and Hbp1 is a transcriptional activator of $p 53$. It is known that p53 acts as both a tumour suppressor and inhibitor of lipogenesis by inhibiting Srebp1c. ${ }^{24} 2531$ Our findings, combined with those of others, indicate that miR-21 plays critical roles in the pathogenesis of NAFLD and HCC. In addition, miR-21 deletion in $p 53$ knockout mice reduced the incidence of liver cancer $;{ }^{37}$ and $p 53$ deletion alone promoted hepatosteatosis, ${ }^{25}$ further supporting the unique role for miR-21 in linking NAFLD to HCC via the Hbp1-p53-Srepb1c pathway. miR-21 significantly inhibits expression of PTEN, ${ }^{34}$ a negative regulator of NASH in mice, ${ }^{38}$ suggesting that it might, in part, mediate the inhibitory effect of miR-21 on hepatosteatosis. In this study, we found that $H b p 1$ is a direct target of miR-21 and confirmed that Hbp1 modulates the inhibitory function of miR-21-ASO on hepatosteatosis and carcinogenesis simultaneously.

We have shown that miR-21 is highly expressed in hepatocytes (figure 1A). Meanwhile, miR-21 is increased but its target Hbp 1 is reduced in livers of HFD-treated mice and human individuals with NAFLD and HCC. All these features of miR-21 lead us to focus on its role in linking NAFLD to HCC. Although other miRNAs might contribute to the development of NAFLD and HCC, few other hepatocyte-specific miRNAs meet the above criteria like miR-21. These include increased expression in both NAFLD and HCC of human and mouse, reduced expression of their target genes in mouse models and human patients, and high expression in hepatocytes. We demonstrated that miR-21 knockdown led to upregulated Hbp1 and $p 53$, downregulated Srebp1c and decreased expression of hepatic Scd1, Gpat and Fasn. These findings are consistent with earlier reports that $p 53$ knockout promoted Srebp1c transcription and in turn increased expression of Scd1, Gpat and Fasn, and subsequently hepatic lipogenesis, ${ }^{25} 28$ suggesting that miR-21-dependent Hbp1-p53 pathway inhibition of Srebp1c transcription represents a candidate pathway to cause NAFLD.

There is evidence to suggest that inhibition of $p 53$ attenuates steatosis and liver injury, ${ }^{39} 40$ which is inconsistent with our findings and those of others. ${ }^{25} 2641$ One possible explanation is that p53 is a negative regulator of hepatic lipid accumulation in the early stages of NAFLD, but $p 53$ is highly expressed at the advanced stages of NAFLD, which may contribute to the high level of apoptosis associated with NASH. In patients with liver steatosis without inflammation, $p 53$ expression was significantly lower than in steatohepatitis, ${ }^{42}$ further suggesting that $\$ 53$ plays different roles in the various developmental stages of NAFLD. Although the mechanism(s) by which miR-21 controls lipogenesis and carcinogenesis in liver clearly requires further investigation, our study has identified an important role for the interaction of miR-21 with Hbp1 in obesity-induced NAFLD and HCC. 
Contributors HW and RN: acquisition of data. XC and CJS: analysis and interpretation of data. GS: obtaining funding, study supervision, study concept and design and drafting of the manuscript.

Funding Supported in part from grants received from the NIDDK R01 (1R01DK102601-01), Minnesota Medical Foundation, NIH Clinical and Translational Science Award at the University of Minnesota (UL1TR000114).

Competing interests None declared.

Ethics approval All procedures involving mice were approved by the Institutional Animal Care Committee at the University of Minnesota, University of California San Francisco, and the Agency for Science Technology and Research Singapore.

Provenance and peer review Not commissioned; externally peer reviewed.

Open Access This is an Open Access article distributed in accordance with the Creative Commons Attribution Non Commercial (CC BY-NC 4.0) license, which permits others to distribute, remix, adapt, build upon this work non-commercially, and license their derivative works on different terms, provided the original work is properly cited and the use is non-commercial. See: http://creativecommons.org/ licenses/by-nc/4.0/

\section{REFERENCES}

1 Yang JD, Roberts LR. Hepatocellular carcinoma: a global view. Nat Rev Gastroenterol Hepatol 2010;7:448-58.

2 Nair S, Mason A, Eason J, et al. Is obesity an independent risk factor for hepatocellular carcinoma in cirrhosis? Hepatology 2003;36:150-5.

3 Calle E, Kaaks R. Overweight, obesity and cancer: epidemiological evidence and proposed mechanisms. Nat Rev Cancer 2004:4:579-91.

4 Bartel D. MicroRNAs: genomics, biogenesis, mechanism, and function. Cell 2004;116:281-97.

5 Esquela-Kerscher A, Slack F. Oncomirs-microRNAs with a role in cancer. Nat Rev Cancer 2006:6:259-69.

6 Cheung 0 , Puri P, Eicken C, et al. Nonalcoholic steatohepatitis is associated with altered hepatic microRNA expression. Hepatology 2008;48:1810-20.

7 Gramantieri L, Fornari F, Callegari E, et al. MicroRNA involvement in hepatocellular carcinoma. J Cell Mol Med 2008;12:2189-204.

8 Esau C, Davis S, Murray SF, et al. miR-122 regulation of lipid metabolism revealed by in vivo antisense targeting. Cell Metab 2006;3:87-98.

9 Ladeiro Y, Couchy G, Balabaud C, et al. MicroRNA profiling in hepatocellular tumors is associated with clinical features and oncogene/tumor suppressor gene mutations. Hepatology 2008:47:1955-63.

$10 \mathrm{Ng} \mathrm{R}, \mathrm{Wu} \mathrm{H}$, Xiao H, et al. Inhibition of microRNA-24 expression in liver prevents hepatic lipid accumulation and hyperlipidemia. Hepatology 2014;60:554-64.

11 Ahrens $\mathrm{M}$, Ammerpohl 0 , von Schönfels $\mathrm{W}$, et al. DNA methylation analysis in nonalcoholic fatty liver disease suggests distinct disease-specific and remodeling signatures after bariatric surgery. Cell Metab 2013;18:296-302.

12 Friedman R, Farh K, Burge C, et al. Most mammalian mRNAs are conserved targets of microRNAs. Genome Res 2009;19:92-105.

13 Krek A, Grün D, Poy $M$, et al. Combinatorial microRNA target predictions. Nat Genet 2005:37:495-500.

14 Yang J-H, Li J-H, Shao P, et al. starBase: a database for exploring microRNA-mRNA interaction maps from Argonaute CLIP-Seq and Degradome-Seq data. Nucleic Acids Res 2011;39(Suppl 1):D202-9

15 Harfe BD, McManus MT, Mansfield JH, et al. The RNaselll enzyme Dicer is required for morphogenesis but not patterning of the vertebrate limb. Proc Natl Acad Sci USA 2005;102:10898-903.

16 Postic C, Magnuson MA. DNA excision in liver by an albumin-Cre transgene occurs progressively with age. Genesis 2000;26:149-50.
17 Mattis AN, Song G, Hitchner K, et al. A screen in mice uncovers repression of lipoprotein lipase by microRNA-29a as a mechanism for lipid distribution away from the liver. Hepatology 2014;61:142-51.

18 Nakai $H$, Fuess S, Storm TA, et al. Unrestricted hepatocyte transduction with adeno-associated virus serotype 8 vectors in mice. J Virol 2005;79:214-24.

19 Vickers KC, Shoucri BM, Levin MG, et al. MicroRNA-27b is a regulatory hub in lipid metabolism and is altered in dyslipidemia. Hepatology 2013;57:533-42.

20 Cui W, Chen SL, Hu K-Q. Quantification and mechanisms of oleic acid-induced steatosis in HepG2 cells. Am J Transl Res 2010;2:95-104.

21 Gomez-Lechon MJ, Donato MT, Martínez-Romero A, et al. A human hepatocellular in vitro model to investigate steatosis. Chem Bio Interact 2007;165:106-16.

22 Li H, Wang W, Liu X, et al. Transcriptional factor HBP1 targets p16INK4A, upregulating its expression and consequently is involved in Ras-induced premature senescence. Oncogene 2010;29:5083-94.

23 Tevosian SG, Shih HH, Mendelson KG, et al. HBP1: a HMG box transcriptional repressor that is targeted by the retinoblastoma family. Genes Dev 1997;11:383-96.

24 Levine AJ, Momand J, Finlay CA. The p53 tumour suppressor gene. Nature 1991;351:453-6

25 Wang $X$, Zhao X, Gao X, et al. A new role of p53 in regulating lipid metabolism. J Mol Cell Biol 2013;5:147-50.

26 Yahagi $\mathrm{N}$, Shimano $\mathrm{H}$, Matsuzaka $\mathrm{T}$, et al. p53 involvement in the pathogenesis of fatty liver disease. J Biol Chem 2004;279:20571-5.

27 Edwards PA, Tabor D, Kast HR, et al. Regulation of gene expression by SREBP and SCAP. Biochim Biophys Acta 2000;1529:103-13.

28 Horton JD, Goldstein JL, Brown MS. SREBPs: activators of the complete program of cholesterol and fatty acid synthesis in the liver. J Clin Invest 2002;109:1125-31.

29 Postic C, Girard J. The role of the lipogenic pathway in the development of hepatic steatosis. Diabetes Metab 2008;34:643-8.

30 Vermeulen K, Van Bockstaele DR, Berneman ZN. The cell cycle: a review of regulation, deregulation and therapeutic targets in cancer. Cell Prolif 2003;36:131-49.

31 Schupp M, Chen F, Briggs ER, et al. Metabolite and transcriptome analysis during fasting suggest a role for the p53-Ddit4 axis in major metabolic tissues. BMC Genomics 2013;14:758.

32 Kawano Y, Cohen DE. Mechanisms of hepatic triglyceride accumulation in non-alcoholic fatty liver disease. J Gastroenterol 2013;48:434-41.

33 Bartlett K, Eaton S. Mitochondrial $\beta$-oxidation. Eur J Biochem 2004;271:462-9.

34 Meng $F$, Henson $R$, Wehbe-Janek $H$, et al. MicroRNA-21 regulates expression of the PTEN tumor suppressor gene in human hepatocellular cancer. Gastroenterology 2007;133:647-58.

35 Becker $\mathrm{P}$, Niesler B, Tschopp 0, et al. MicroRNAs as mediators in the pathogenesis of non-alcoholic fatty liver disease and steatohepatitis. Z Gastroenterol 2014;52:1-27.

36 Krichevsky AM, Gabriely G. miR-21: a small multi-faceted RNA. J Cell Mol Med 2009;13:39-53.

37 Ma X, Choudhury SN, Hua $X$, et al. Interaction of the oncogenic miR-21 microRNA and the p53 tumor suppressor pathway. Carcinogenesis 2013;34:1216-23.

38 Watanabe S, Horie Y, Suzuki A. Hepatocyte-specific Pten-deficient mice as a nove model for nonalcoholic steatohepatitis and hepatocellular carcinoma. Hepatol Res 2005;33:161-6.

39 Castro RE, Ferreira D, Afonso MB, et al. miR-34a/SIRT1/p53 is suppressed by ursodeoxycholic acid in the rat liver and activated by disease severity in human non-alcoholic fatty liver disease. J Hepatol 2013;58:119-25.

40 Derdak Z, Villegas KA, Harb R, et al. Inhibition of p53 attenuates steatosis and live injury in a mouse model of non-alcoholic fatty liver disease. $J$ Hepatol 2013;58:785-91

41 Jiang $P$, Du $W$, Wang $X$, et al. p53 regulates biosynthesis through direct inactivation of glucose-6-phosphate dehydrogenase. Nat Cell Biol 2011;13:310-16.

42 Panasiuk A, Dzieciol J, Panasiuk B, et al. Expression of $\mathrm{p53}$, Bax and $\mathrm{BCl}-2$ proteins in hepatocytes in non-alcoholic fatty liver disease. World J Gastroenterol 2006;12:6198. 